\title{
Effect of segmented telescope phasing errors on adaptive optics performance
}

\author{
Marcos A. van Dam ${ }^{\mathrm{a}}$, Sam Ragland ${ }^{\mathrm{b}}$, and Peter L. Wizinowich ${ }^{\mathrm{b}}$ \\ ${ }^{a}$ Flat Wavefronts, 21 Lascelles Street, Christchurch 8022, New Zealand \\ ${ }^{\mathrm{b}}$ W. M. Keck Observatory, 65-1120 Mamalahoa Highway, Kamuela, HI 96743, USA
}

\begin{abstract}
The largest existing optical telescopes and the next generation of extremely large telescopes are all segmented telescopes. The primary mirror segments must be phased in order to attain the diffraction limit of the telescope. In this paper, we investigate the effect of phasing errors on image quality delivered by the adaptive optics systems at the W. M. Keck Observatory using both simulations and on-sky data. We show that a ShackHartmann wavefront sensor is able to measure moderate phasing errors, which can be partially corrected by the deformable mirror. Phase retrieval using multiple short exposure images at and near the focal plane leads to phase maps that are consistent with telescope segment phasing errors of around $150 \mathrm{~nm}$. In the future, we would like to use the phase maps to correct for the segment phasing errors.
\end{abstract}

Keywords: segment phasing, W.M. Keck Observatory, adaptive optics, phase retrieval, phase diversity, ShackHartmann

\section{INTRODUCTION}

The largest existing optical telescopes and the next generation of extremely large telescopes are all segmented telescopes. With the exception of the Giant Magellan Telescope, these segmented telescopes all use tesselated hexagons with small segment gaps. In order to attain the diffraction limit of the telescope, we need to properly phase the segments and correct the atmospheric turbulence with adaptive optics (AO). A question that is seldom addressed is the interaction between telescope segment phasing errors and the image quality delivered by an AO system. In this paper, we investigate the effect of phasing errors on image quality at the W. M. Keck Observatory using both simulations and on-sky data.

Figure 1 shows a narrowband H-band image $(1.65 \mu \mathrm{m})$ taken with AO correction while guiding on a bright natural guide star (NGS) in favourable observing conditions. While the correction is excellent, the point spread function (PSF) images on this target exhibit a quasi-static structure in the first diffraction ring. In this paper,
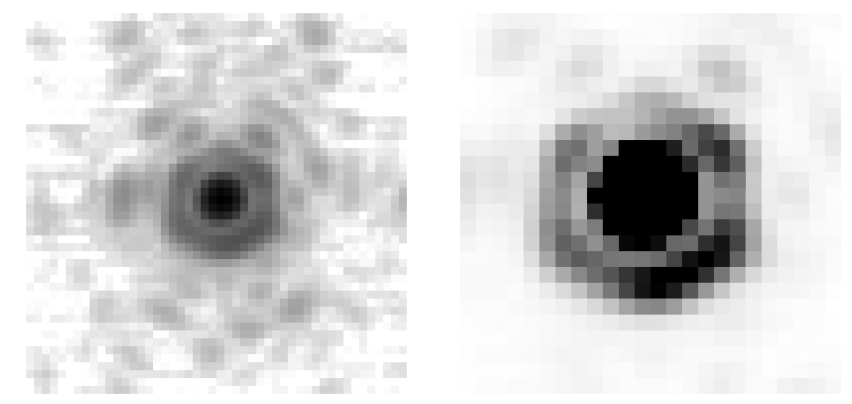

Figure 1: Narrowband H-band image with an asinh stretch (left) and a linear stretch (right) to accentuate the first diffraction ring.

we show how phasing errors can lead to such artefacts and we present results from experiments to recover the phasing errors using images taken with the science camera.

Further author information: send correspondence to Marcos van Dam: marcos@flatwavefronts.com 
Starting in 2002, there was a strong push to understand, characterize and improve the performance of the Keck II AO system. ${ }^{1}$ Hardware upgrades in 2007 and improvements in the calibration and operating software have narrowed the gap between the expected and delivered Strehl ratio. ${ }^{2}$ There is also an ongoing effort at the W. M. Keck Observatory to predict the PSF of the science images based on telemetry from the AO system. ${ }^{4}$ Since the quasi-static artefacts do not appear in the telemetry, they need to be measured and included in the PSF reconstruction algorithm. Currently, this occurs by making long-exposure phase diversity measurements on a bright star at the start of the observation. ${ }^{4}$ Rampy et al investigated the presence of quasi-static aberrations in the AO-corrected images and suspected that telescope segment phasing errors could explain the some of the structure in the PSFs. ${ }^{3}$

The remainder of the paper is distributed as follows. Section 2 introduces the Keck telescopes along with the phasing and AO systems. In Section 3, we show that a Shack-Hartmann wavefront sensor (SH WFS) is able to measure phase discontinuities within a subaperture. End-to-end simulations of the Keck AO system, including phasing errors, are presented in Section 4. In Section 5, we use in-focus and out-of-focus images of the guide star to measure quasi-static aberrations, which are found to be consistent with phasing errors. Concluding remarks are offered in Section 6.

\section{KECK TELESCOPES, SEGMENT PHASING AND ADAPTIVE OPTICS}

In this section, we briefly describe the telescope, followed by the segment phasing, the AO systems and the science instruments.

\subsection{Telescopes}

The W. M. Keck Observatory consists of two almost identical telescopes situated on the summit of Maunakea, Hawaii, at an elevation of $4145 \mathrm{~m}$. The primary mirror of each telescope has 36 hexagonal segments, and a gap between adjacent segments of the order of $3 \mathrm{~mm}$. The distance between opposite vertices of the hexagon is $1.8 \mathrm{~m}$, leading to an effective diameter of $10 \mathrm{~m}$ and a circumscribed diameter of $10.95 \mathrm{~m}$. An image of the telescope pupil is shown in Figure 2.

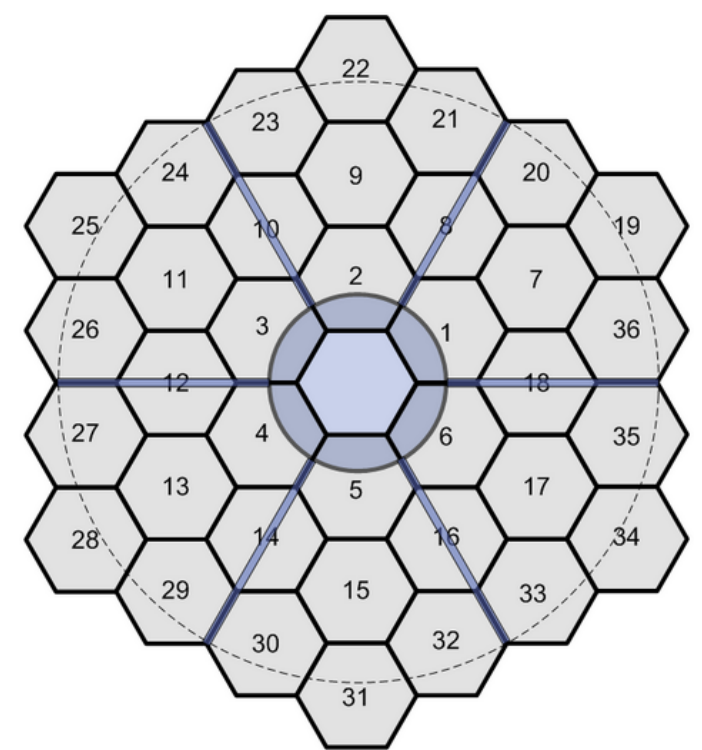

Figure 2: Telescope pupil, including the numbered primary mirror segments, secondary mirror central obscuration and the spiders. The dotted circle represents a circular aperture with a $9 \mathrm{~m}$ diameter. 


\subsection{Phasing camera system}

The Keck telescope uses a visible light phasing camera system (PCS) to phase the segments. ${ }^{5}$ PCS consists of 78 circular subapertures that span adjacent segments, as shown in Figure 3.

The coherence of the images formed by these subapertures are compared with templates to yield estimates of the phase difference between the segments. The broadband phasing algorithm is currently in use ${ }^{5}$ as the narrowband algorithm originally proposed for fine phasing appears to have larger systematic errors. ${ }^{6} 78$ mea-

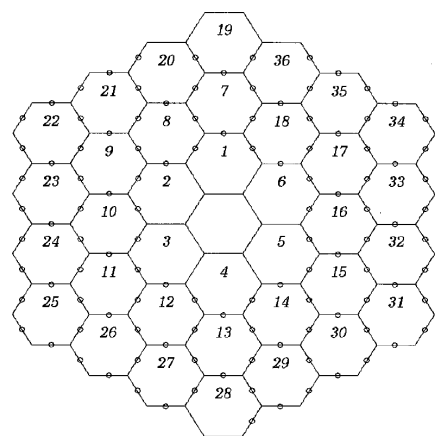

Figure 3: Location of the PCS circular subapertures. ${ }^{5}$ The subapertures between adjacent segments are used for phasing, while the subpertures on the outside are used for registration purposes.

surements are made of the phase difference between pairs of adjacent segment edges to produce estimates of the 36 segment piston terms. The repeatability of the measurement is a surface error of $30 \mathrm{~nm}$, which corresponds to a wavefront error of $60 \mathrm{~nm}$. The accuracy of the measurements, incorporating systematic errors, is claimed to be within $100 \mathrm{~nm}$ of surface (200 nm of wavefront). ${ }^{5}$ In the remainder of the paper, all the segment phasing errors are quoted as wavefront, and not surface, errors.

Telescope phasing occurs approximately every two months, following a segment exchange, where some of the primary mirror segments are removed to be recoated and replaced by freshly coated ones. Once the telescope is phased, the output of the optically phased segments are used to define the reference points of the segment capacitive edge sensors. The active mirror control system (ACS) uses these capacitive sensors to maintain the optical figure of the segmented primary mirror under the changing influences of gravity and temperature. ${ }^{7}$ In addition, a look-up table is used to remove the effect of so-called terrace mode, caused by distortion of the edge sensors under gravity. Without this correction, ACS would impose a terrace-like structure on the primary mirror. The size of the effect is about $3 \mathrm{~nm}$ per degree of elevation. A second look-up tablr corrects for primary mirror focus mode. In an attempt to keep the sensor readings constant, the ACS then imposes a terrace-like structure on the primary mirror. The performance of ACS in maintaining the optical phase with varying gravity vectors, temperature and humidity is understood. However, the performance of with time over time scales of hours to days has not been studied.

Other techniques can be used to phase segmented telescopes by comparing the phase at the edges of adjacent edges. Two such methods proposed for the James Webb Space Telescope have been sky-tested at the W. M. Keck Observatory. Shi et al implemented a dispersed fringe sensor to test fine phasing, ${ }^{8}$ while Albanese et al used a dispersed Hartmann sensor to test coarse phasing. ${ }^{9}$

Methods for phasing from images are investigated in Section 5.

\subsection{Adaptive optics and science instruments}

Each telescope is equipped with essentially identical AO systems. ${ }^{1,2,15,16}$ There are notable differences between the sodium lasers and associated launch optics, ${ }^{10,11}$ and the fact that Keck I has an infrared tip-tilt sensor ${ }^{12,13}$ in addition to a visible light one. Three near infrared science instruments operate behind the AO systems. The Keck I AO system feeds OSIRIS, an integral field spectrograph and imager. On Keck II, the AO-corrected light is directed to NIRSPEC, a high-resolution spectrograph, or NIRC2, an imaging camera. The work reported here uses NIRC2 on Keck II, but the results are equally applicable to the other science instruments. The AO system can guide on NGSs (both point sources and very extended sources, such as Uranus) and laser guide stars (LGSs). 
The same SH WFS, set to the appropriate conjugate altitude, is used in both NGS and LGS mode. It has $20 \times 20,0.562 \mathrm{~m}$ square subapertures across the telescope primary mirror. The detector is an $80 \times 80$ pixel CCD-39 which is typically read as a quad cell with $2 \times 2$ pixel binning. A quad cell, which consists of $2 \times 2$ pixels per subaperture, is used as it limits the noise on the centroid caused by the detector read-out noise. The use of a quad cell, however, leads to an uncertain relationship between the raw centroid (in units of pixels) and the angular displacement of the spots (in arcseconds). The relationship between the two is called the centroid gain and depends on the size and shape of the spot on the WFS. ${ }^{14}$ Calibrating the centroid gain while operating on the sky is a well-known but difficult to solve problem. The main implications of using quad cells on this work is that the calibrated reference centroids (also known as centroid offsets or origins) are not perfectly compensated on the sky, since the size of the spots is variable and only approximately known. This means that a significant fraction of the calibrated non-common path aberrations leak onto the science image in a time-varying, unpredictable manner. The dominant non-common path aberration on Keck II is $300 \mathrm{~nm}$ of $90^{\circ}$ astigmatism on the science path (Figure 4) which is corrected using reference centroids on the WFS. As a result, we expect to see astigmatism on the images if the centroid gain is not correctly set.

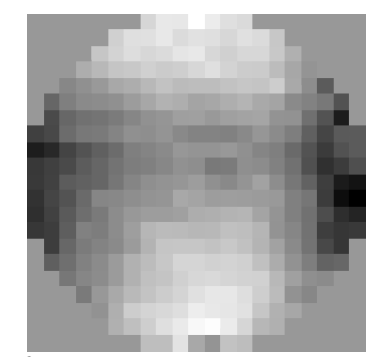

Figure 4: Typical voltages applied on the deformable mirror during calibrations to compensate for non-common path aberrations.

Two separate mirrors are used to correct the wavefront: tip-tilt mirror and a deformable mirror (DM). The DM is 349 actuator mirror made by Xinetics. More information on the AO systems can be found in Wizinowich et $a .^{15}$

\section{RESPONSE OF WAVEFRONT SENSORS TO PHASING ERRORS}

It is commonly believed that because the SH WFS is a geometric WFS, it is insensitive to discontinuities in the wavefront. Here, we investigate the response of the SH WFS to phase discontinuities using a one-dimensional toy example. In Section 4, we present full end-to-end simulations of the Keck AO system.

Consider a single, one-dimensional subaperture with a phase iscontinuity in the middle of the subaperture, as shown in Figure 5. For small phase discontinuities, the centroid from the resulting image is exactly the same
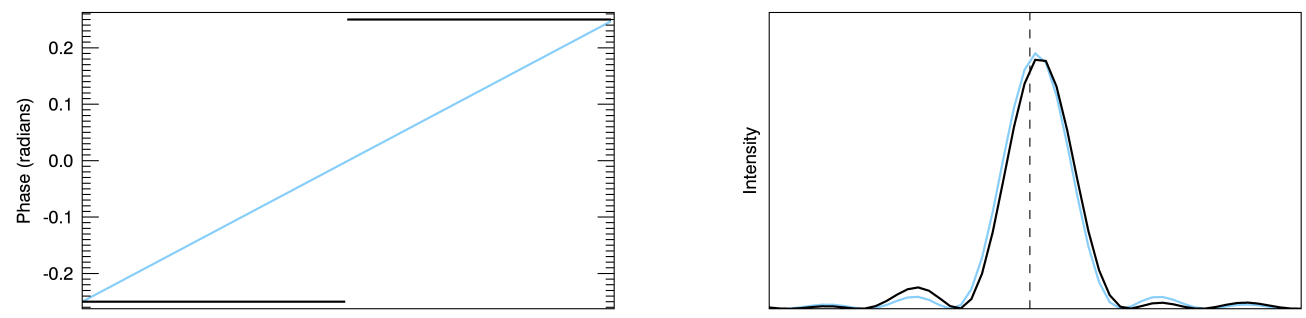

Figure 5: Phase discontinuity across a subaperture and a phase with a constant tilt (left). These two phases at the pupil produce images (right) with the same centroids. The dotted line denotes the center of the detector.

as from a pure tilt phase with the same values at the edges of the subaperture. As the magnitude of the phases discontinuity increases, the response becomes non-linear, as shown in Figure 6. It can be seen that the quad 


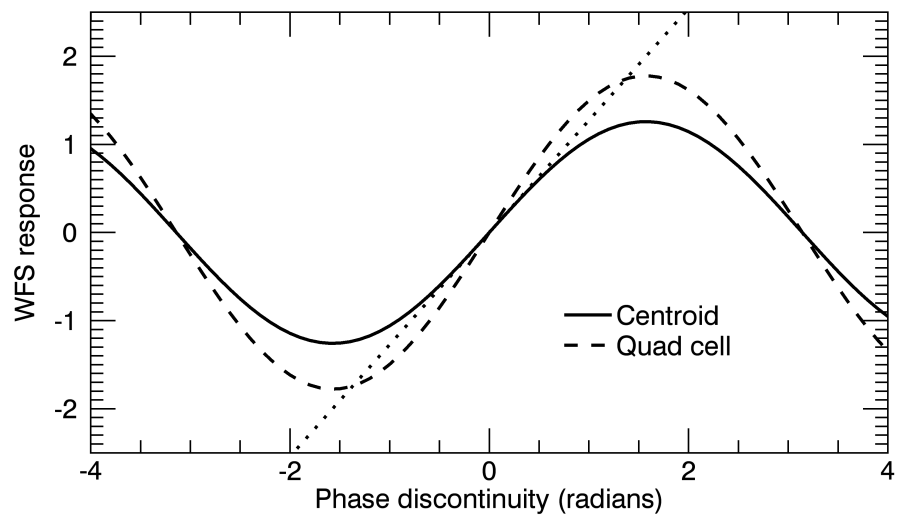

Figure 6: Response of the SH WFS using the centroid and the quad cell to a phase discontinuity across the subaperture. The straight dotted line represents the reponse of the centroid to a tip-tilt.

cell is even more sensitive to discontinuities than the centroid is, and for this ideal case, the dynamic range is high. If the discontinuity is greater than $\lambda / 2$, however, then the centroid estimate will have the wrong sign. This clearly shows the potential for an AO system based on a SH WFS to measure and partially correct phasing errors, provided that they are sufficiently small.

\section{END-TO-END SIMULATIONS OF PHASING ERRORS}

In this section, we explain how we generate synthetic telescope segment phasing errors. We describe the end-toend simulations written in yao, ${ }^{17}$ and report the simulation results.

\subsection{Simulating segment piston errors}

An optical phase measurement is made between each pair of neighboring segments. Let us assume that the measurement is a Gaussian process with zero mean, and each measurement is uncorrelated with every other measurement. The error in the measurement could stem from many sources, such as measurement noise, atmospheric turbulence, errors in the mirror figure, segment tip-tilt, etc. Here, we are not concerned with the source of the error. We generate an interaction matrix, $M$, that relates how poking each of the 36 segments affects the vector of 78 phase measurements between adjacent segments. The reconstructor, $R$, is the pseudo-inverse of $M$ and converts phase measurements into segment piston estimates. The interaction and reconstructor matrices are displayed in Figure 7. The eigenmodes of the interaction matrix are shown in Figure 8. It can be seen from the
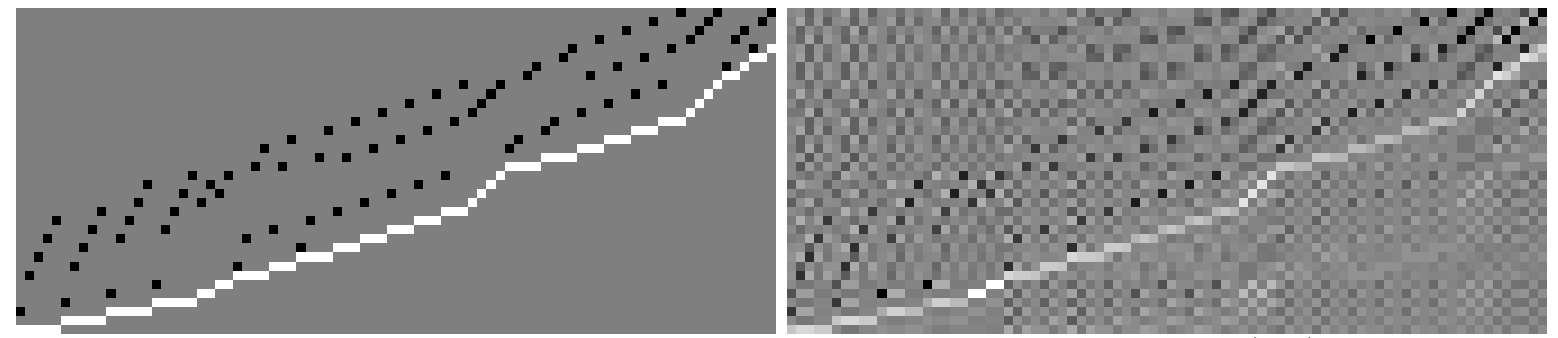

Figure 7: Interaction matrix between segment piston and the phase measurements (left) and the associated reconstruction matrix (right).

eigenmodes that the dominant modes are low-order modes, so we expect that most of the power in the segment phasing error will reside at low spatial frequencies. The telescope phasing errors were simulated by multiplying the reconstructor by a vector of 78 random numbers from a zero-mean Gaussian distribution. If the error in the phase measurements between adjacent segments has a standard deviation of $100 \mathrm{~nm}$, then the resulting segment 


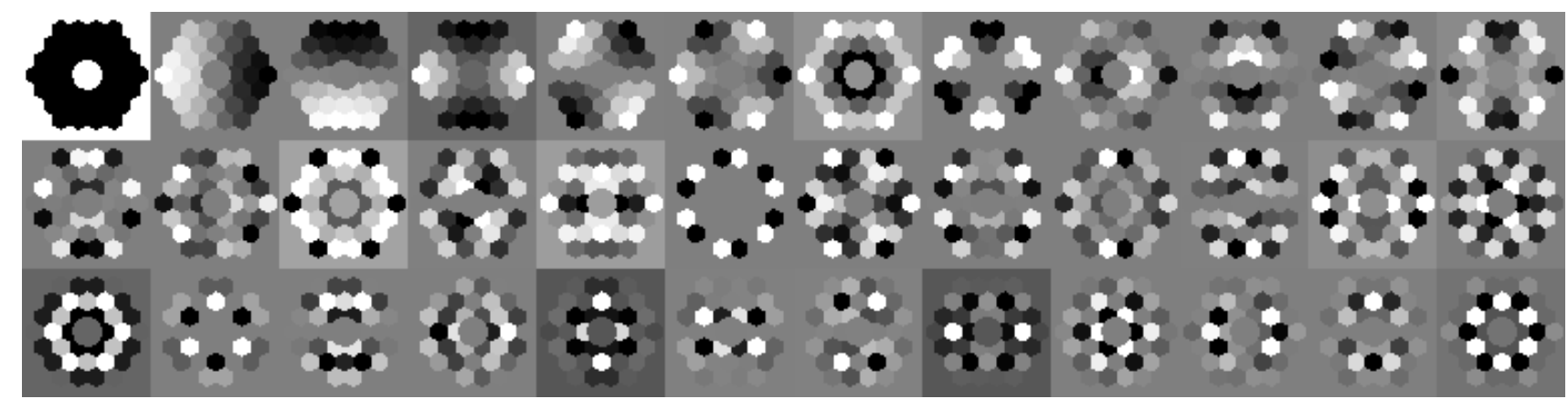

Figure 8: Eigenmodes of the interaction matrix between segment piston and the phase measurements.

piston error is $69 \mathrm{~nm}$, the same value calculated by Chanan et $\left.a l^{5}\right)$. The resulting phase across the pupil tends to be low-order, as shown in Figure 9. We do not believe that the figure of the primary mirror looks like the
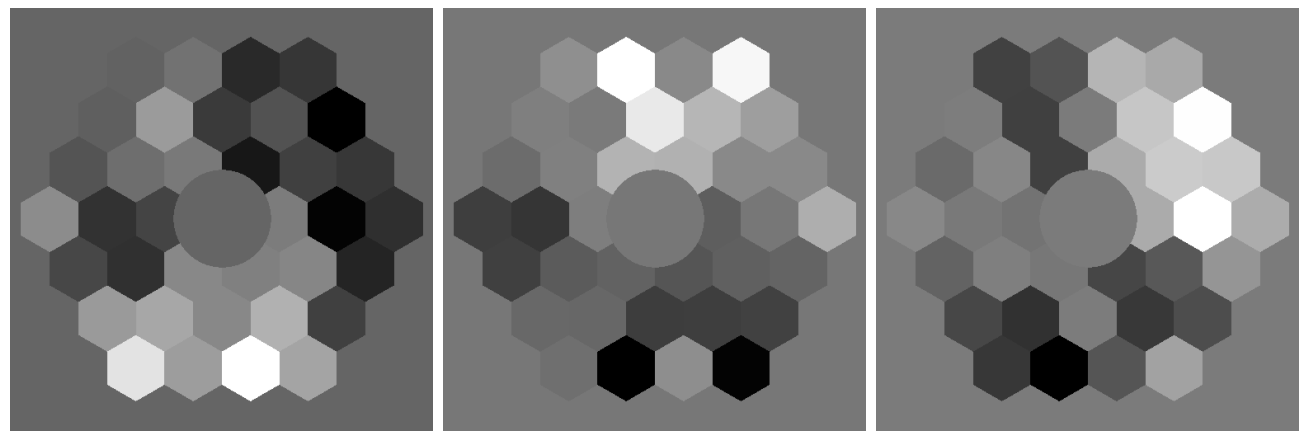

Figure 9: The three sets of random piston errors used in the simulations.

images in Figure 9: the wavefront error is likely to be far more complex, with high spatial frequencies due to figure errors and segment tip-tilt. Nevertheless, the point is that random errors in measuring the phase difference between segments lead to mostly low-order aberrations in the primary mirror figure. The questions to address is to what extent are these phase errors measured by the wavefront sensor and corrected.

\subsection{Description of simulations}

End-to-end Monte Carlo simulations were run using yao to understand how phasing errors on the Keck telescopes affect the PSF delivered by the AO system. Yao is a flexible, open-source, user-configurable simulation platform written in the yorick language by François Rigaut, with contributions from a number of users. ${ }^{17}$ Custom yorick scripts were written to simulate the specifics of the Keck telescopes and AO systems, such as the telescope pupil, DM actuator locations and wavefront reconstructor.

The simulations used $500 \times 500$ pixels over a $11.2 \mathrm{~m}$ diameter telescope pupil, with each pixel spanning $0.0224 \mathrm{~m}$. The WFS has $20 \times 20$ subapertures, each with $2 \times 22.94^{\prime \prime}$ pixels, operating with an $m_{R}=6$ guide star at a single wavelength $640 \mathrm{~nm}$. Using broadband light would improve the fidelity of the simulations. The effect of charge diffusion ${ }^{1}$ of the CCD is modeled as a convolution by a Gaussian with a FWHM of $0.6^{\prime \prime}$. The non-common path aberrations, and hence the reference centroids, were assumed to be zero.

The tip-tilt mirror is modeled as an ideal mirror with no dynamics. The DM has 349 actuators in the same configuration as the real DM. An actuator wavefront of $0.4 \mu \mathrm{m}$ per Volt is assumed, with a cross-talk between adjacent actuators of $15 \%$. The voltage is restricted to $\pm 10 \mathrm{~V}$ The restriction in voltage difference between adjacent actuators and the actuator hysteresis are not modeled.

A two frame loop delay was used for both the tip-tilt and DM loops. The reconstructor and control laws are the same as implemented on the sky for a bright guide star. The tip-tilt controller was 


$$
H_{T T}[z]=\frac{0.35}{1-0.6 z^{-1}-0.32 z^{-2}-0.08 z^{-3}},
$$

while the DM controller was

$$
H_{D M}[z]=\frac{0.5}{1-0.999 z^{-1}} .
$$

21080 iterations were run at a frame rate of $1054 \mathrm{~Hz}$ for $20 \mathrm{~s}$ of simulated data.

The atmospheric parameters used in these simulations are derived from a typical Mauna Kea profile and are reproduced in Table 1. The value of $r_{0}$ is set to $0.15 \mathrm{~m}$ at $500 \mathrm{~nm}$ and an infinite outer scale is used in the simulations. The guide star and the science target are assumed to be the same object and located at zenith.

Table 1: Turbulence profile used in the simulations

\begin{tabular}{rrrr}
\hline Elevation $(\mathrm{m})$ & Turbulence fraction & Wind speed $\left(\mathrm{m} \mathrm{s}^{-1}\right)$ & Wind direction $\left(^{\circ}\right)$ \\
\hline 0 & 0.517 & 10 & 0 \\
500 & 0.119 & 10 & 45 \\
1000 & 0.063 & 10 & 172 \\
2000 & 0.061 & 10 & 237 \\
4000 & 0.105 & 10 & 336 \\
8000 & 0.081 & 10 & 99 \\
16000 & 0.054 & 10 & 277 \\
\hline
\end{tabular}

\subsection{Simulation results}

Simulations were run with varying amounts of the three realizations of phasing errors shown in Figure 9. The average results are presented in Table 2. For segment piston errors with an RMS value $\leq 75 \mathrm{~nm}$, the additional

Table 2: H-band Strehl ratio of AO corrected images with varying degrees of phasing error. The additional wavefront error due to the phasing error is computed using the Maréchal approximation.

\begin{tabular}{rrrrrrrr}
\hline RMS phasing error $(\mathrm{nm})$ & 0 & 50 & 75 & 100 & 125 & 150 & 175 \\
\hline H-band Strehl ratio & 0.733 & 0.729 & 0.724 & 0.708 & 0.672 & 0.603 & 0.512 \\
Additional wavefront error (nm) & 0 & 19 & 29 & 49 & 77 & 116 & 157 \\
\hline
\end{tabular}

error is small because the WFS can measure it and the DM can partially correct it. However, once the phasing errors reach $150 \mathrm{~nm}$, the errors are amplified because the WFS measurements can have the wrong sign. Recall that the WFS can accurately measure phase discontinuities as large as $\approx \lambda / 3(210 \mathrm{~nm})$, and that the sign of the WFS measurement is wrong for discontinuities greater than $\lambda / 2(320 \mathrm{~nm})$.

This finding can be visualized by inspecting Figure 10, which plots the PSFs on a linear scale for different amounts of segment piston. Clear structure in the first diffraction ring can be see for $150 \mathrm{~nm}$ or more of phasing error. In this example, the phasing error on the left of Figure 9 was used. Simulations were run with $150 \mathrm{~nm}$ of segment piston error to see what the residuals wavefront is. The average residual wavefront was saved and is displayed in Figure 11 for two cases: with and without atmospheric turbulence. The AO system only corrects a small fraction of the segment piston, and there is significant low spatial frequency power that the DM could correct if only the WFS could measure it.

\section{ESTIMATING THE SEGMENT PHASING ERRORS FROM IMAGES}

In this section, we present the use of images at or near the focal plane to estimate the segment phasing error. We describe previous work on this topic, and show new results using NIRC2 images. 


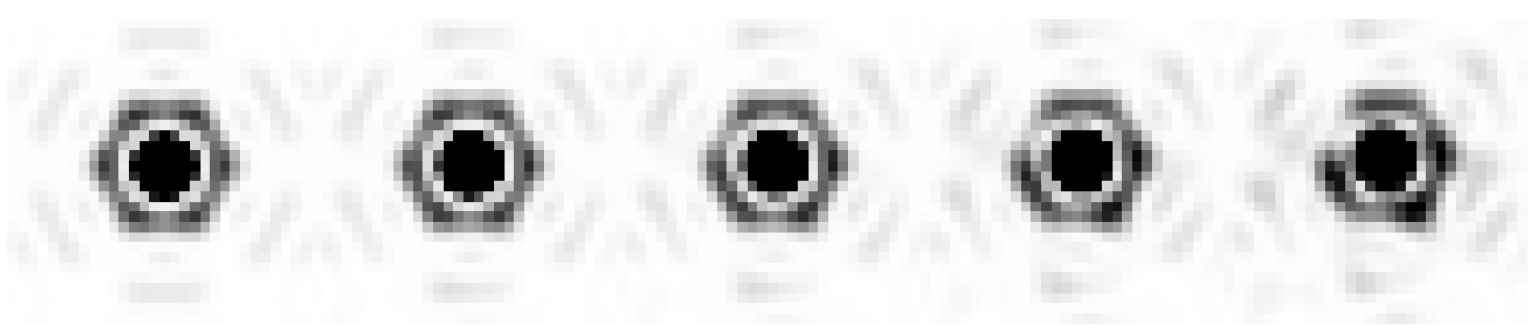

Figure 10: H-band PSFs delivered by the AO system for, from left to right, $0 \mathrm{~nm}, 100 \mathrm{~nm}, 125 \mathrm{~nm}, 150 \mathrm{~nm}$ and $175 \mathrm{~nm}$ of segment piston error. The PSFs are plotted on a linear scale.
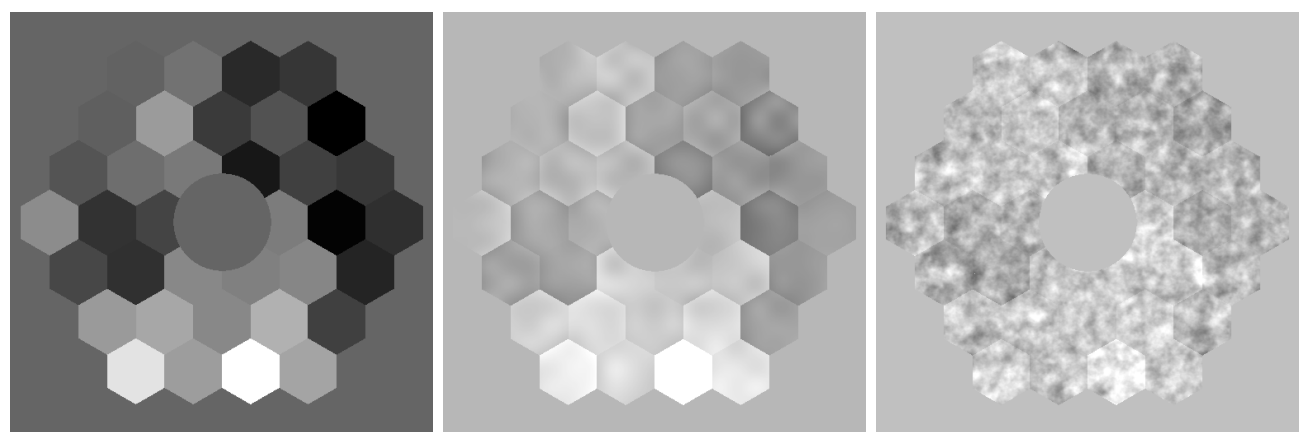

Figure 11: Applied segment piston error (left), residual wavefront without turbulence (center) and with turbulence (right).

\subsection{Previous work}

Two experiments based on images taken at or near the focal plane have been previously employed to measure the telescope phasing error.

Löfdahl et al took simultaneous in-focus and out-of-focus short exposure images of a bright star at $900 \mathrm{~nm}$ with a CCD. ${ }^{19}$ Unfortunately, the wavefront errors due to atmospheric turbulence were too strong, and they were unable to retrieve the phase and thereby measure segment piston. The authors postulated that better seeing $\left(r_{0} \geq 0.3 \mathrm{~m} !\right)$ or AO-correction would be required. Alternatively, the measurement could be made at longer wavelengths using an eAPD array.

Another method for phasing, called phase discontinuity sensing (PDS), was developed by Chanan et al and enjoyed success. This consists of imaging a bright star at $3.3 \mu \mathrm{m}$. The method was initially developed for the NIRC camera on Keck I, and was later extended to use the NIRC2 camera on Keck II. ${ }^{18,20}$ Severely defocused long-exposure images are taken successively on both sides of focus. The efficiency and performance of PDS can be improved by correcting the wavefront with $\mathrm{AO}$ and using shorter wavelengths (probably K-band). Unfortunately, the WFS can only be sufficiently defocused in one direction. The performance of PDS with a single image needs to be investigated further.

\subsection{Phase retrieval from in-focus NIRC2 images}

Images of HIP 1602, with an apparent magnitude of $m_{V}=6.78$, were taken on 15 October 2016 using NIRC2, first in focus and then at four different defocus planes. The AO loops were closed on the target itself. In this section, we present the results based on the in-focus images, while an analysis of the defocus images is presented in Section 5.3. In all cases, the filter is Brackett Gamma, a narrowband filter with a central wavelength of $2.168 \mu \mathrm{m}$.

50 consecutive frames were saved with an exposure time of $8 \mathrm{~ms}$, and a time lag between successive frames of $3.2 \mathrm{~s}$. Many frames are needed to average out the effect of the residual atmospheric turbulence. The structure in the reduced images, shown in Figure 12, varies from frame to frame.

A modified Gerchberg-Saxton algorithm ${ }^{21}$ was used to recover the wavefront error based on the short exposure images. The individual wavefront error maps are shown in Figure 13. There is a structure in the wavefront error 


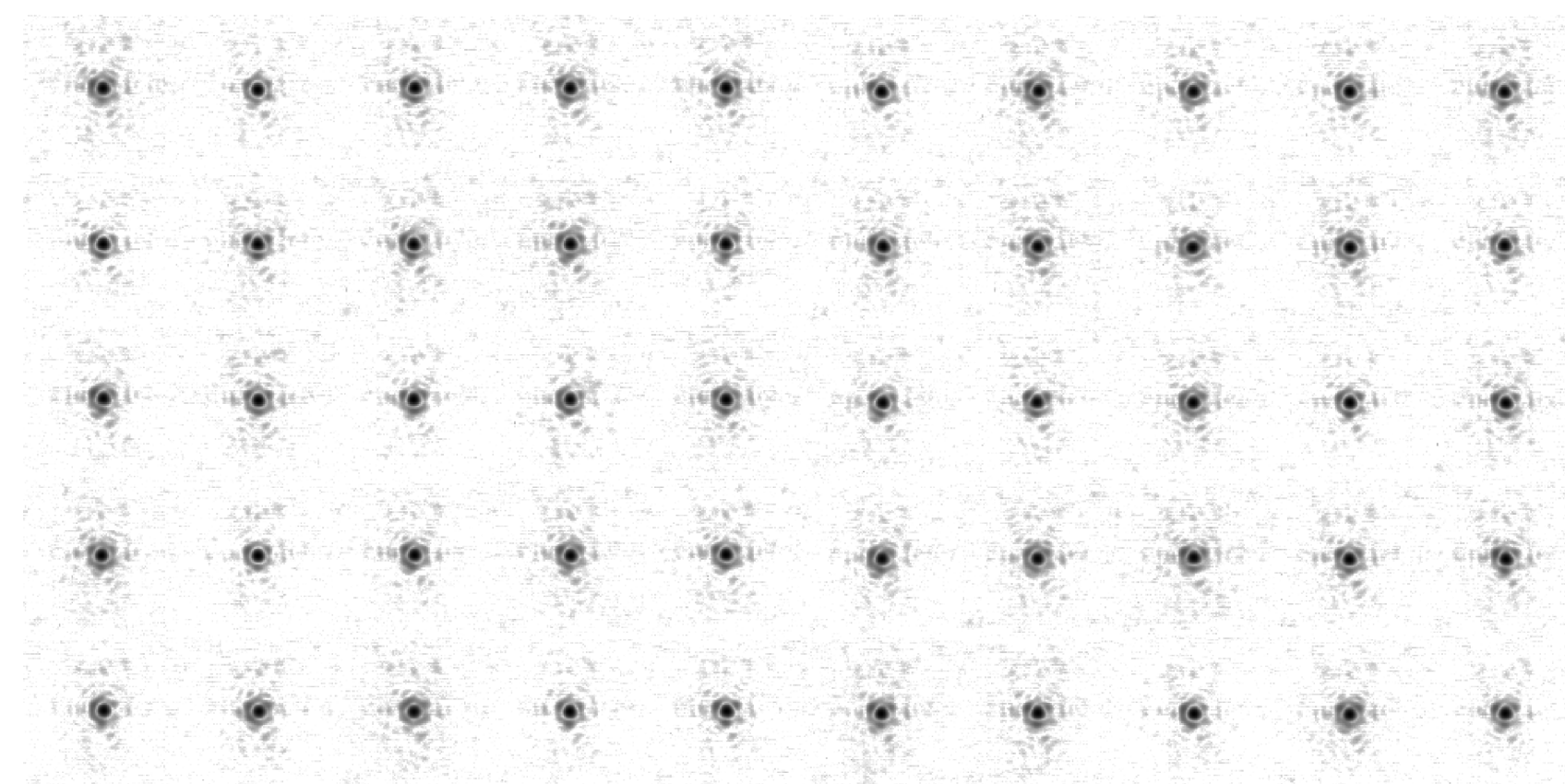

Figure 12: In focus short-exposure PSFs.

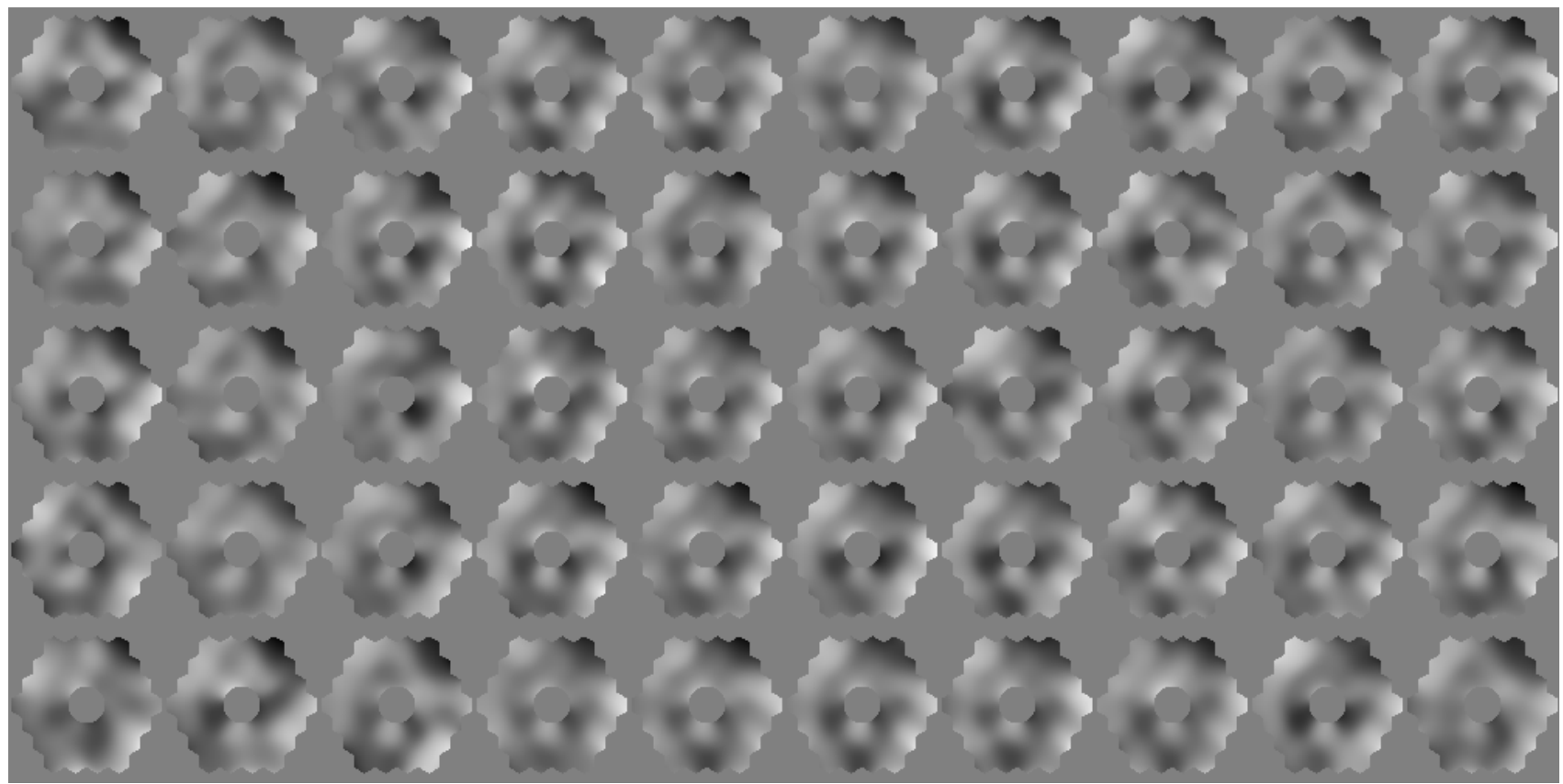

Figure 13: Individual phase estimates based on the short exposure PSFs.

that is common to all of the short-exposure images. The images at the focal plane suffer from phase ambiguity: it can be shown that, assuming uniform amplitude at the pupil plane, the phase $\phi(x, y)$ leads to the same images as $-\phi(-x,-y)$. The two possibilities for the average reconstructed wavefront are displayed in Figure 14. Defocused images are used in Section 5.3 to break the phase ambiguity and determine that the phase on the left correct. The astigmatism term in the reconstructed phase maps is small, which shows that the astigmatism corrected by the reference centroids has not leaked into the image via incorrect centroid gains. The structure in the phase maps is consistent with segment phasing errors. 

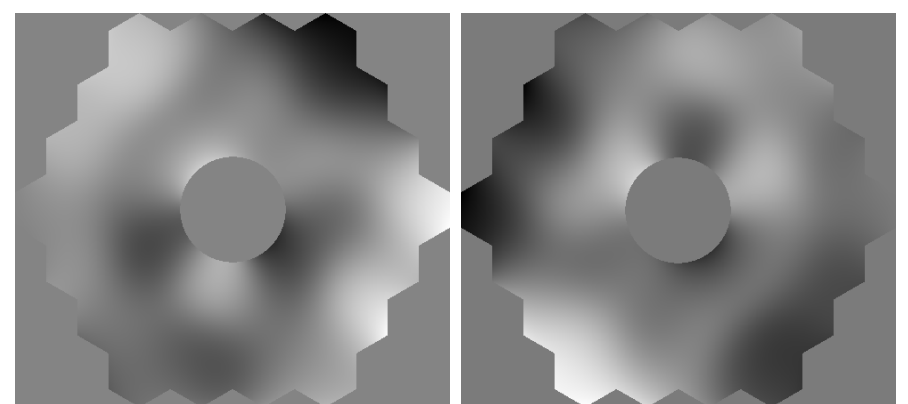

Figure 14: Average phase maps retrieved from all 50 short-exposure images. Both phase maps are consistent with the in-focus images, but the reconstructions from defocused images show that the phase map on the left is correct.

\subsection{Phase retrieval from phase-diverse NIRC2 images}

The same measurements were also made using out-of-focus images. The images were taken by defocusing the WFS by $\pm 3 \mathrm{~mm}$, thereby adding a focus wavefront error of approximately $\pm 540 \mathrm{~nm}$ RMS. 50 images were captured on either side of focus, with sample images shown in Figure 15. We can immediately see that the level of defocus

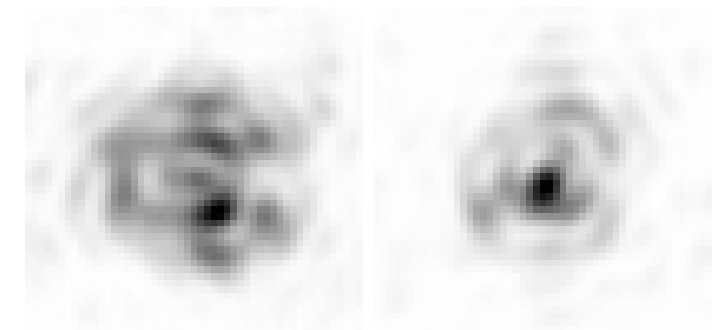

Figure 15: Short exposure images with $-3 \mathrm{~mm}$ (left) and $3 \mathrm{~mm}$ (right) of defocus.

is not the same, with the image on the left appearing to be more out of focus than the image on the right, indicating that the calibrated focus position was wrong. This focus term was removed from the reconstruction.

There are two ways to combine the multiple images at the three different focus positions to obtain a phase estimate: by estimating the phase corresponding to each image and then averaging, or by doing a joint estimate of the phase given all of the images. We present the results from both methods here.

First, the phase was estimated for each image using a modified Gerchberg-Saxton algorithm similar to the one used to calibrate the non-common path error of the AO system. ${ }^{21}$ The phase ambiguity problem is eliminated by forcing the solution to be consistent with data from all three focal positions (Figure 16) (left). The wavefront error has mostly high-spatial frequency structure and an RMS value of $111 \mathrm{~nm}$.

The second way to retrieve the phase is to simultaneously solve for the phase that minimizes the difference between all of the measured images and the modeled images. The open source software package yorick-opra was used for this purpose. ${ }^{22}$ The first 28 Zernike modes were reconstructed, with the reconstruction displayed in Figure 16 (right). The reconstruction looks similar and the RMS wavefront was almost identical at $112 \mathrm{~nm}$. The reconstruction is consistent with the phase found from the in-focus images in Figure 14, although the use of defocused images improves the reconstruction of high spatial frequencies.

A third set of data was captures with the WFS stage $\pm 12 \mathrm{~mm}$ out of focus. This is the maximum distance that the focus stage can move in the negative direction. The Gerchberg-Saxton code had difficulties due to phase wrapping, but the yorick-opra code ran well and returned an identical phase map to the one shown in Figure 16.

The true phasing errors are likely larger, because phase retrieval algorithms are not able to capture the high spatial frequencies that result from phase discontinuities. By inspection of Table 2, we find that a residual error of $112 \mathrm{~nm}$ stems from around $145 \mathrm{~nm}$ of phasing error. 

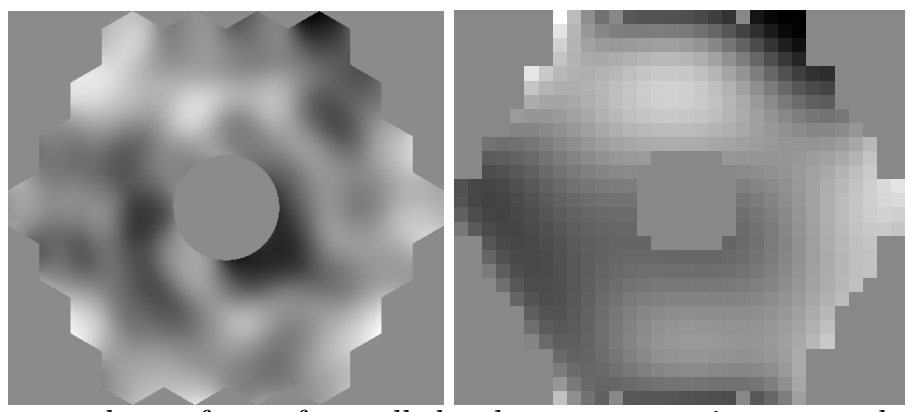

Figure 16: Average reconstructed wavefronts from all the short-exposure images at the three different focal positions using the modified Gerchberg-Saxton algorithm (left) and OPRA (right). The Gerchberg-Saxton algorithm estimates the phase pixel by pixel, while OPRA reconstructs the first 28 Zernike modes.

\section{CONCLUSION AND DISCUSSION}

In this paper, we investigate the effect of segment telescope phasing errors on the image quality delivered by an AO system. In particular, we are interested in knowing whether telescope phasing errors could be responsible for the artefacts in the PSF of NIRC2 images delivered by the Keck II AO system.

Using a toy example and full end-to-end simulations, we show that a SH WFS is able to measure phase discontinuities of up to $\approx \lambda / 3$, and the $\mathrm{DM}$ is able to partially correct them. For larger phase discontinuities, the sign of the WFS measurements can be wrong and exacerbate the phasing errors.

Short-exposure images were taken on the sky. Using phase-diverse phase retrieval, we show that the recovered phase maps, with an RMS value of about $110 \mathrm{~nm}$, are consistent with telescope phasing errors of around $150 \mathrm{~nm}$. This leads to a relative reduction in $\mathrm{H}$ - and $\mathrm{K}$-band Strehl ratios of $28 \%$ and $18 \%$ respectively.

There is still a lot of work to be done on this topic. The WFS simulations could be expanded to include broadband light, LGSs, and other WFSs, such as a pyramid WFS. We could also use the output phase maps to update the reference centroids of the WFS or, ideally, the reference of the capacitive sensors in the telescope active mirror control system in order to correct for the piston errors.

\section{Acknowledgments}

The authors wish to recognize and acknowledge the very significant cultural role and reverence that the summit of Maunakea has always had within the indigenous Hawaiian community. We are most fortunate to have the opportunity to conduct observations from this mountain. The authors would like to thank Mats Löhfdahl and Agustí Pintó for useful correspondence regarding details of prior work on their respective phasing experiments, and Mitchell Troy, Gary Chanan, Richard Cohen and Kuochou Tai for providing valuable information about the phasing system.

\section{REFERENCES}

[1] M. A. van Dam, D. Le Mignant and B. A. Macintosh, "Performance of the Keck Observatory Adaptive-Optics System," Appl. Opt. 43, 5458-5467 (2004).

[2] E. M. Johansson et al, "Upgrading the Keck AO wavefront controllers," Proc SPIE 7015, 70153E (2008).

[3] R. Rampy et al., "Understanding and correcting low order residual static aberrations in adaptive optics corrected images," SPIE Astronomical Telescopes + Instrumentation, 91485I (2014).

[4] S. Ragland et al, "Point spread function determination for Keck adaptive optics," Proc. SPIE 99091, 99091P (2016).

[5] G. Chanan et al., "Phasing the mirror segments of the Keck telescopes: the broadband phasing algorithm," Appl. Opt. 37 140-155 (1998).

[6] G. Chanan et al., "Phasing the mirror segments of the Keck telescopes II: the narrowband phasing algorithm," Appl. Opt. 39, 4706-4714 (2000). 
[7] R. W. Cohen, T. S. Mast and J. E. Nelson, "Performance of the W.M. Keck telescope active mirror control system," Proc. SPIE 2199, 105 (1994).

[8] Fang Shi et al., "Experimental verification of dispersed fringe sensing as a segment phasing technique using the Keck telescope," Appl. Opt. 43, 4474-4481 (2004).

[9] M. Albanese et al., "Verification of the James Webb Space Telescope Coarse Phase Sensor Using the Keck Telescope," Proc. SPIE 6265, 62650Z (2006).

[10] J. Chin et al, "Laser guide star facility developments at W. M. Keck Observatory," Proc. SPIE 9148, 914808 (2014).

[11] J. Chin et al, "Keck II laser guide star adaptive optics system and performance with the TOPTICA/MPBC laser," Proc. SPIE 9909, 99090S (2016).

[12] P. Wizinowich et al, "A near-infrared tip-tilt sensor for the Keck I laser guide star adaptive optics system," Proc. SPIE 9148 91482B (2014).

[13] B. Femenia-Castella et al, "Status and new developments with the Keck I near-infrared tip-tilt sensor," Proc. SPIE bf 9909, 990925 (2016).

[14] M. A. van Dam, "Measuring the centroid gain of a ShackHartmann quad-cell wavefront sensor by using slope discrepancy," JOSA A 22, 1509-1514 (2005).

[15] P. L. Wizinowich et al, "The WM Keck Observatory laser guide star adaptive optics system: overview," PASP 118, 297 (2006).

[16] M. A. van Dam et al, "The WM Keck Observatory laser guide star adaptive optics system: performance characterization," PASP 118, 310 (2006).

[17] F. Rigaut and M. van Dam, "Simulating Astronomical Adaptive Optics Systems Using Yao," AO4ELT3, 13173 (2013).

[18] G. Chanan, M. Troy, and E. Sirko, "Phase discontinuity sensing: a method for phasing segmented mirrors in the infrared," Applied Optics 38, 704-713 (1999).

[19] M. G. Lofdahl et al, "Phase diversity experiment to measure piston misalignment on the segmented primary mirror of the Keck II telescope," SPIE Astronomical Telescopes + Instrumentation, 1190-1201 (1998).

[20] Agusti Pinto, "PDS2 users manual," August 17, 2005.

[21] P. Atcheson, D. S. Acton, and P. A. Lightsey, "Instrument-level phase retrieval wavefront sensing and correction for astronomical telescopes," In Astronomical Telescopes and Instrumentation, Proc. SPIE, 228239 (2003).

[22] D. Gratadour, F. Rigaut, and B. Neichel, "Tomographic phase diversity for non-common path aberrations retrieval on wide field AO systems," 2nd AO4ELT conference-Adaptative Optics for Extremely Large Telescopes (2011). 